\title{
Simplified fractional Fourier transforms
}

\author{
Soo-Chang Pei and Jian-Jiun Ding \\ Department of Electrical Engineering, National Taiwan University, Taipei, Taiwan
}

Received February 25, 2000; revised manuscript received May 31, 2000; accepted August 1, 2000

\begin{abstract}
The fractional Fourier transform (FRFT) has been used for many years, and it is useful in many applications. Most applications of the FRFT are based on the design of fractional filters (such as removal of chirp noise and the fractional Hilbert transform) or on fractional correlation (such as scaled space-variant pattern recognition). In this study we introduce several types of simplified fractional Fourier transform (SFRFT). Such transforms are all special cases of a linear canonical transform (an affine Fourier transform or an $A B C D$ transform). They have the same capabilities as the original FRFT for design of fractional filters or for fractional correlation. But they are simpler than the original FRFT in terms of digital computation, optical implementation, implementation of gradient-index media, and implementation of radar systems. Our goal is to search for the simplest transform that has the same capabilities as the original FRFT. Thus we discuss not only the formulas and properties of the SFRFT's but also their implementation. Although these SFRFT's usually have no additivity properties, they are useful for the practical applications. They have great potential for replacing the original FRFT's in many applications. (c) 2000 Optical Society of America [S0740-3232(00)01812-3]

OCIS codes: $070.2580,070.2590,070.6020,070.6110$.
\end{abstract}

\section{INTRODUCTION}

The fractional Fourier transform ${ }^{1,2}$ (FRFT) is a generalization of the conventional Fourier transform. It is defined as

$$
\begin{aligned}
O_{F}^{\alpha}[f(t)]= & \left(\frac{1-j \cot \alpha}{2 \pi}\right)^{1 / 2} \exp \left(\frac{j}{2} u^{2} \cot \alpha\right) \\
& \times \int_{-\infty}^{\infty} \exp \left(-j u t \csc \alpha+\frac{j}{2} t^{2} \cot \alpha\right) f(t) \mathrm{d} t .
\end{aligned}
$$

When $\alpha=\pi / 2$, the FRFT becomes the conventional Fourier transform; when $\alpha=0$, it is the same as the identity operation. It also satisfies the additivity property

$$
O_{F}^{\alpha}\left\{O_{F}^{\beta}[f(t)]\right\}=O_{F}^{\beta}\left\{O_{F}^{\alpha}[f(t)]\right\}=O_{F}^{\alpha+\beta}[f(t)] .
$$

The properties of the FRFT were described in Refs. 1 and 2 . The FRFT has been popular in recent years and useful in many applications such as optical system analysis, filter design, solving differential equations, phase retrieval, and pattern recognition.

The linear canonical transform (LCT) is a further generalization of the FRFT. ${ }^{3,4}$ It is defined as

$$
\begin{aligned}
& O_{F}^{(a, b, c, d)}[f(t)]=\left(\frac{1}{j 2 \pi b}\right)^{1 / 2} \exp \left(\frac{j}{2} \frac{d}{b} u^{2}\right) \\
& \times \int_{-\infty}^{\infty} \exp \left(-\frac{j}{b} u t+\frac{j}{2} \frac{a}{b} t^{2}\right) f(t) \mathrm{d} t \\
& b \neq 0, b \neq 0 \\
& O_{F}^{(a, 0, c, d)}[f(t)]=(d)^{1 / 2} \exp \left(\frac{j}{2} c d u^{2}\right) f(\mathrm{~d} u) \quad b=0
\end{aligned}
$$

$$
a d-b c=1
$$

must be satisfied to meet the requirement of the energyconservation law. Abe and Sheridan call the LCT the special affine Fourier transform, ${ }^{5}$ and Bernardo ${ }^{6}$ calls it the $A B C D$ transform. The LCT satisfies the matrix property as

$$
O_{F}^{\left(a_{2}, b_{2}, c_{2}, d_{2}\right)}\left\{O_{F}^{\left(a_{1}, b_{1}, c_{1}, d_{1}\right)}[f(t)]\right\}=O_{F}^{(e, f, g, h)}[f(t)],
$$

where

$$
\left[\begin{array}{ll}
e & f \\
g & h
\end{array}\right]=\left[\begin{array}{ll}
a_{2} & b_{2} \\
c_{2} & d_{2}
\end{array}\right]\left[\begin{array}{ll}
a_{1} & b_{1} \\
c_{1} & d_{1}
\end{array}\right] .
$$

Many operations are special cases of a LCT. When $\{a, b, c, d\}=\{\cos \alpha, \sin \alpha,-\sin \alpha, \cos \alpha\}$, the LCT becomes a FRFT of order $\alpha{ }^{7}$ When $\{a, b, c, d\}$ $=\{1, z \lambda / 2 \pi, 0,1\}$ the LCT becomes a Fresnel transform (describes monochromatic light with wavelength $\lambda$ propagating through free space for a distance $z$ ). When $\{a, b, c, d\}=\{1,0, \tau, 1\}$ the LCT becomes a chirpmultiplication operation. And, when $\{a, b, c, d\}$ $=\left\{\sigma^{-1}, 0,0, \sigma\right\}$, the LCT becomes a scaling operation.

The digital implementation of a LCT can be written as

$$
\begin{aligned}
Y_{(a, b, c, d)}(m)= & (j 2 \pi b)^{-1 / 2} \Delta \exp \left(\frac{j}{2} \frac{d}{b} m^{2} \Delta_{u}^{2}\right) \\
& \times \sum_{n=-N}^{N} \exp \left(-\frac{j}{b} n m \Delta_{u} \Delta_{t}\right. \\
& \left.+\frac{j}{2} \frac{a}{b} n^{2} \Delta_{t}^{2}\right) y(n),
\end{aligned}
$$

where

$$
\begin{array}{r}
y(n)=f\left(n \Delta_{t}\right), \quad Y_{(a, b, c, d)}(m)=F_{(a, b, c, d)}\left(m \Delta_{u}\right), \\
n, m=-N,-N+1, \ldots, N .
\end{array}
$$


In Ref. 8 we suggest that we can choose the sampling intervals as

$$
\Delta_{t} \Delta_{u}=2 \pi b /(2 N+1),
$$

and then Eq. (8) can be rewritten as

$$
\begin{aligned}
Y_{(a, b, c, d)}(m)= & (j 2 \pi b)^{-1 / 2} \Delta_{t} \exp \left(\frac{j}{2} \frac{d}{b} m^{2} \Delta_{u}^{2}\right) \\
& \times \sum_{n=-N}^{N} \exp \left(-\frac{j 2 \pi m n}{2 N+1}+\frac{j}{2} \frac{a}{b} n^{2} \Delta_{t}^{2}\right) \\
& \times y(n) .
\end{aligned}
$$

Then we need only one fast Fourier transform to implement the LCT. Specifically, for a FRFT,

$$
\begin{aligned}
Y_{\alpha}(m)= & \left(\frac{1-j \cot \alpha}{2 \pi}\right)^{1 / 2} \Delta_{t} \exp \left(\frac{j}{2} m^{2} \cot \alpha \Delta_{u}^{2}\right) \\
& \times \sum_{n=-N}^{N} \exp \left(-\frac{j 2 \pi m n}{2 N+1}+\frac{j}{2} n^{2} \cot \alpha \Delta_{t}^{2}\right) \\
& \times y(n) \\
\Delta_{t} \Delta_{u}= & 2 \pi \sin \alpha /(2 N+1)
\end{aligned}
$$

Details of the digital implementation of the FRFT-LCT combination were discussed in Refs. 8 and 9. The complexity of computation for LCT is $2 P+(P / 2) \log _{2} P$, where $P=2 N+1$ is the number of sampling points. This is so because we need two $P$-point multiplication operations and one $P$-point fast Fourier transform.

We can use other ways to implement the FRFT-LCT. Usually we need three optical components (lenses and free spaces) to do this. For the gradient-index (GRIN) medium system we can use a GRIN medium to implement the scaled FRFT (not the ordinary FRFT), but when we want to implement other LCT's we must use other components. For the radar system we can use an emitter, a receiver, and free space to implement the LCT when $b$ $\leqslant 0$.

In this paper we introduce some simplified forms of the fractional Fourier transform, which we call the simplified fractional Fourier transforms (SFRFT's). As was discussed in Ref. 10, when the FRFT is used for fractional correlation with optical implementation, the chirp term $\exp \left(j \cot \alpha u^{2} / 2\right)$ of the FRFT in Eq. (1) can be removed. This is just one type of SFRFT. In this paper we try to find SFRFT's that have the same capabilities as the original FRFT for the design of fractional filters or for fractional correlation and at the same time are simplest for digital computation, optical implementation, GRIN medium implementation, and radar system implementation. These SFRFT's will have great potential to substitute for the original FRFT's in many applications.

In this paper we discuss the following topics:

(1) The conditions in which the LCT will have the same effects as the FRFT for design of a fractional filter.

(2) The type 1 SFRFT. It produces the same results as the FRFT in the design of fractional filters but is simpler in digital implementation. Although for optical implementation a SFRFT of type 1 is not so simple as one of type 2 , we need merely to adjust one optical component for a different value of $\alpha$.

(3) The type 2 SFRFT. It has the same effects as the FRFT in the design of fractional filters but its optical implementation is simpler.

(4) SRFT's of types 3 and 4. They have the same effects as FRFT's is for the design of fractional filters but their implementation for a GRIN medium or a radar system is simpler.

(5) The canonical correlation, which is the generalization of fractional correlation.

(6) The type 5 SFRFT. It has the same effects as FRFT for fractional correlation but is simpler in digital implementation.

\section{SFRFT WITH THE SAME CAPABILITIES FOR FILTER DESIGN AS THE ORIGINAL FRFT}

\section{A. Conditions for Similar Capabilities of LCT and FRFT}

Many of the applications of the FRFT, such as removing chirp noise $^{11}$ and as a fractional Hilbert transform, ${ }^{12}$ are based on the design of a fractional filter. The fractional filter is the special case of fractional convolution: ${ }^{13}$

$$
O_{\text {conv }}^{\alpha}[x(t), y(t)]=O_{F}^{-\alpha}\left\{O_{F}^{\alpha}[x(t)] O_{F}^{\alpha}\{y[(t)]\} .\right.
$$

The formula for the fractional filter system can be written as

$$
z(t)=O_{F}^{-\alpha}\left\{O_{F}^{\alpha}[x(t)] H(u)\right\},
$$

where $x(t)$ is the input, $z(t)$ is the output, and $H(u)$ is the transfer function of the filter. In fact, we can replace the FRFT with the LCT, generalize the fractional convolution into the canonical convolution,

$$
\begin{aligned}
O_{\mathrm{conv}}^{(a, b, c, d)} & {[x(t), y(t)] } \\
= & O_{F}^{(d,-b,-c, a)}\left\{O_{F}^{(a, b, c, d)}[x(t)] O_{F}^{(a, b, c, d)}[y(t)]\right\},
\end{aligned}
$$

and generalize the fractional filter system into a canonical filter system,

$$
z(t)=O_{F}^{(d,-b,-c, a)}\left\{O_{F}^{(a, b, c, d)}[x(t)] H(u)\right\} .
$$

Although there are three free parameters for the canonical filter design (four parameters, $a, b, c$, and $d$, and one constraint, $a d-b c=1$ ), but for Ref. 14 we used the Wigner distribution to illustrate that only the value

$$
b / a
$$

will have the appropriate effects on the results of canonical convolution and thus on the design of the canonical filter. We can illustrate this in another way: Suppose that

$$
\begin{aligned}
& z_{1}(t)=O_{F}^{\left(d_{1},-b_{1},-c_{1}, a_{1}\right)}\left\{O_{F}^{\left(a_{1}, b_{1}, c_{1}, d_{1}\right)}[x(t)] H_{1}(u)\right\}, \\
& z_{2}(t)=O_{F}^{\left(d_{2},-b_{2},-c_{2}, a_{2}\right)}\left\{O_{F}^{\left(a_{2}, b_{2}, c_{2}, d_{2}\right)}[x(t)] H_{2}(u)\right\} .
\end{aligned}
$$

Then, after some calculation, we obtain 


$$
\begin{aligned}
z_{1}(t)= & \frac{1}{j 2 \pi b_{1}} \exp \left(-\frac{j}{2} \frac{a_{1}}{b_{1}} t^{2}\right) \int_{-\infty}^{\infty} \exp \left[\frac{j}{b_{1}} u(t-k)\right] \\
& \times H_{1}(u) \mathrm{d} u \int_{-\infty}^{\infty} \exp \left(\frac{j}{2} \frac{a_{1}}{b_{1}} k^{2}\right) x(k) \mathrm{d} k, \\
z_{2}(t)= & \frac{1}{j 2 \pi b_{2}} \exp \left(-\frac{j}{2} \frac{a_{2}}{b_{2}} t^{2}\right) \int_{-\infty}^{\infty} \exp \left[\frac{j}{b_{2}} u(t-k)\right] \\
& \times H_{2}(u) \mathrm{d} u \int_{-\infty}^{\infty} \exp \left(\frac{j}{2} \frac{a_{2}}{b_{2}} k^{2}\right) x(k) \mathrm{d} k
\end{aligned}
$$

In the case that

$$
\frac{a_{1}}{b_{1}}=\frac{a_{2}}{b_{2}}, \quad b_{1}, b_{2} \neq 0,
$$

we can prove that, if

$$
H_{2}(u)=H_{1}\left(b_{1} u / b_{2}\right),
$$

then

$$
\begin{aligned}
z_{2}(t)= & \frac{1}{j 2 \pi b_{2}} \exp \left(-\frac{j}{2} \frac{a_{1}}{b_{1}} t^{2}\right) \int_{-\infty}^{\infty} \exp \left[\frac{j}{b_{2}} u(t-k)\right] \\
& \times H_{1}\left(\frac{b_{1}}{b_{2}} u\right) \mathrm{d} u \int_{-\infty}^{\infty} \exp \left(\frac{j}{2} \frac{a_{1}}{b_{1}} k^{2}\right) x(k) \mathrm{d} k \\
= & \frac{1}{j 2 \pi b_{1}} \exp \left(-\frac{j}{2} \frac{a_{1}}{b_{1}} t^{2}\right) \int_{-\infty}^{\infty} \exp \left[\frac{j}{b_{1}} u(t-k)\right] \\
& \times H_{1}(s) \mathrm{d} s \int_{-\infty}^{\infty} \exp \left(\frac{j}{2} \frac{a_{1}}{b_{1}} k^{2}\right) x(k) \mathrm{d} k \\
= & z_{1}(t) .
\end{aligned}
$$

That is, when we design the canonical filter we can use the LCT with the parameters $\left\{a_{2}, b_{2}, c_{2}, d_{2}\right\}$ to obtain the same results as when we use the parameters $\left\{a_{1}, b_{1}, c_{1}, d_{1}\right\}$ when $a_{1} / b_{1}=a_{2} / b_{2}$. We need only to scale the original transfer function by a factor of $b_{2} / b_{1}$. This leads to the conclusion that what can be done with a filter with a LCT that has parameters $\left\{a_{2}, b_{2}, c_{2}, d_{2}\right\}$ can also be done with a filter with a LCT that has parameters $\left\{a_{1}, b_{1}, c_{1}, d_{1}\right\}$ when $a_{1} / b_{1}=a_{2} / b_{2}$. Thus, although there are four parameters for the LCT, for the design of the the canonical filter only the value of $a / b$ will have the same effects. If two LCT's have the same value of $a / b$, then for purposes of filter design they are in fact the same.

For a FRFT of order $\alpha$,

$$
a / b=\cot \alpha .
$$

Thus all the LCT's with parameters $\{a, b, c, d\}$ will have the same effects as the FRFT of order $\alpha$ for design of a canonical filter if $a / b=\cot \alpha$. In this section we find, for each type of implementation, what values of $\{a, b, c, d\}$ will satisfy $a / b=\cot \alpha$ and have the smallest implementation costs. Then a LCT with this set of parameters can replace the FRFT with order $\alpha$ for this type of implementation, and it is the SFRFT that we want.

\section{B. Type 1 SFRFT}

The digital implementation of a LCT is described by Eqs. (10) and (11). To design the SFRFT we can choose

$$
\left.b=1 \quad \text { (fixed value of } \Delta_{t} \Delta_{u}\right),
$$

$d=0 \quad$ (saving one chirp-multiplication operation),

$$
a / b=\cot \alpha,
$$

and the parameters of the LCT become

$$
\left[\begin{array}{ll}
a & b \\
c & d
\end{array}\right]=\left[\begin{array}{cc}
\cot \alpha & 1 \\
-1 & 0
\end{array}\right] .
$$

Then we can define the first type of SFRFT as follows:

\section{Type 1 SFRFT}

$$
\begin{aligned}
O_{F(1)}^{\alpha}[f(t)]= & (j 2 \pi)^{-1 / 2} \\
& \times \int_{-\infty}^{\infty} \exp \left(-j u t+\frac{j}{2} t^{2} \cot \alpha\right) f(t) \mathrm{d} t,
\end{aligned}
$$

and its inverse is

$$
\begin{aligned}
O_{I F(1)}^{\alpha}\left[F_{\alpha}(u)\right]= & \left(\frac{j}{2 \pi}\right)^{1 / 2} \exp \left(-\frac{j}{2} u^{2} \cot \alpha\right) \\
& \times \int_{-\infty}^{\infty} \exp (j u t) F_{\alpha}(u) \mathrm{d} t .
\end{aligned}
$$

In a special case of LCT for which $\{a, b, c, d\}$ $=\{0,-1,1,-\cot \alpha\}$, we can prove that

$$
O_{I F(1)}^{\alpha}\left\{O_{F(1)}^{\alpha}[f(t)]\right\}=f(t) \quad \text { for all } \alpha .
$$

A type 1 SFRFT has the same effect as a FRFT of order $\alpha$ for filter design, but for digital implementation it is simpler than the original FRFT. After substituting $\{a, b, c, d\}=\{\cot \alpha, 1,-1,0\}$ into Eq. (11) we find that we can implement the type 1 SFRFT as

$$
\begin{aligned}
Y_{\alpha}(m)= & (j 2 \pi)^{-1 / 2} \Delta_{t} \sum_{n=-N}^{N} \exp \left(-j \frac{2 \pi m n}{2 N+1}\right. \\
& \left.+\frac{j}{2} n^{2} \cot \alpha \Delta_{t}^{2}\right) y(n),
\end{aligned}
$$

where

$$
\begin{aligned}
& y(n)=f\left(n \Delta_{t}\right), \quad Y_{\alpha}(m)=F_{\alpha}\left(m \Delta_{u}\right), \\
& n, m=-N,-N+1, \ldots, N, \quad \Delta_{t} \Delta_{u}=2 \pi /(2 N+1),
\end{aligned}
$$

and the inverse SFRFT can be implemented as

$$
\begin{aligned}
y(n)= & \left(\frac{j}{2 \pi}\right)^{1 / 2} \frac{1}{(2 N+1) \Delta_{t}} \exp \left(-\frac{j}{2} n^{2} \cot \alpha \Delta_{t}^{2}\right) \\
& \times \sum_{m=-N}^{N} \exp \left(j \frac{2 \pi m n}{2 N+1}\right) Y_{\alpha}(m) .
\end{aligned}
$$

In comparison with the digital implementation of the original FRFT in Eq. (12), here one- $P$ point $(P=2 N$ +1 ) chirp-multiplication operation is saved. This is so 
because we choose $d=0$. So the complexity of digital implementation is reduced from $2 P+(P / 2) \log _{2} P$ to $P$ $+(P / 2) \log _{2} P$. The type $1 \mathrm{SFRFT}$ has a little less complexity than the original FRFT for digital implementation, but the performance for filter design remains the same.

Besides, there is another advantage of the type 1 SFRFT. We note that the value of $\Delta_{t} \Delta_{u}=2 \pi /(2 N$ + 1) will not vary with $\alpha$. For the original FRFT, because $\Delta_{t} \Delta_{u}=2 \pi \sin \alpha /(2 N+1)$, we must use different sampling values of $\Delta_{t}$ or $\Delta_{u}$ for different values of $\alpha$. This problem does not exist for a type 1 SFRFT.

Conventional convolution requires only one integration operation, but fractional convolution ${ }^{13,15,16}$ usually requires three integrations. Now we can use the type 1 SFRFT to define the fractional convolution:

$$
z(t)=O_{I F(1)}^{\alpha}\left\{O_{F(1)}^{\alpha}[x(t)] O_{F(1)}^{\alpha}[y(t)]\right\}
$$

then

$$
\begin{aligned}
z(t)= & j^{-1 / 2}(2 \pi)^{-3 / 2} \exp \left(-\frac{j}{2} t^{2} \cot \alpha\right) \int_{-\infty}^{\infty} \exp (j u t) \\
& \times \int_{-\infty}^{\infty} \exp \left(-j u k+\frac{j}{2} k^{2} \cot \alpha\right) x(k) \mathrm{d} k \\
& \times \int_{-\infty}^{\infty} \exp \left(-j u s+\frac{j}{2} s^{2} \cot \alpha\right) y(s) \mathrm{d} s \mathrm{~d} u \\
= & (j 2 \pi)^{-1 / 2} \exp \left(-\frac{j}{2} t^{2} \cot \alpha\right) \int_{-\infty}^{\infty} \int_{-\infty}^{\infty} \delta(t-k-s) \\
& \times \exp \left[\frac{j}{2}\left(k^{2}+s^{2}\right) \cot \alpha\right] x(k) y(s) \mathrm{d} s \mathrm{~d} k \\
= & (j 2 \pi)^{-1 / 2} \exp \left(-\frac{j}{2} t^{2} \cot \alpha\right) \\
& \times \int_{-\infty}^{\infty} \exp \left\{\frac{j}{2}\left[k^{2}+(t-k)^{2}\right] \cot \alpha\right\} \\
& \times x(k) y(t-k) \mathrm{d} s \mathrm{~d} k \\
& (j 2 \pi)^{-1 / 2} \int_{-\infty}^{\infty} \exp [j k(k-t) \cot \alpha] \\
& \times x(k) y(t-k) \mathrm{d} s \mathrm{~d} k,
\end{aligned}
$$

and we require only one integration operation. So the fractional convolution defined by the type 1 SFRFT is much simpler than the original convolution. The fractional convolution defined here can also be rewritten as

$$
\begin{aligned}
z(t)= & \exp \left(-\frac{j}{2} \cot \alpha t^{2}\right) \\
& \operatorname{Conv}\left[\exp \left(\frac{j}{2} t^{2} \cot \alpha\right) x(t), \exp \left(\frac{j}{2} t^{2} \cot \alpha\right) y(t)\right],
\end{aligned}
$$

where Conv( ) means the conventional convolution operation.
The type 1 SFRFT also has some advantages for optical implementation. Before discussing that, we first discuss the optical implementation of a LCT and of a filter designed by the LCT. ${ }^{17}$

When we use the optical system with three components to implement the LCT, we can do so by using one of the following two methods. The first method is illustrated in Fig. 1, for which $f_{1}, f_{2}, d_{0}$ are

$$
f_{1}=\frac{k b}{(1-a)}, \quad d_{0}=k b, \quad f_{2}=\frac{k b}{(1-d)},
$$

where $k=2 \pi / \lambda$ is the wave number. Specifically, for a FRFT,

$$
f_{1}=f_{2}=\frac{k \sin \alpha}{(1-\cos \alpha)}, \quad d_{0}=k \sin \alpha .
$$

In as much as the length of free space must be positive, the constraint on our using Fig. 1 to implement the LCT is that

$$
b>0 \text {. }
$$

The second method is illustrated in Fig. 2, where for which the values of $d_{1}, d_{2}$, and $f_{0}$ are

$$
d_{1}=\frac{k(d-1)}{c}, \quad f_{0}=-\frac{k}{c}, \quad d_{2}=\frac{k(a-1)}{c},
$$

and, for a FRFT,

$$
d_{1}=d_{2}=k(\csc \alpha-\cot \alpha), \quad f_{0}=k \csc \alpha .
$$

The lengths of the free space must be positive, so, if we want to use Fig. 2 to implement the LCT, the parameters of the LCT must satisfy one of these conditions:

$$
\begin{aligned}
& a \geqslant 1, \quad d \geqslant 1, \quad b>0 ; \\
& a=d=1, \quad b=0 ; \\
& a \leqslant 1, \quad d \leqslant 1, \quad a d<1, \quad b>0 ; \\
& a \leqslant 1, \quad d \leqslant 1, \quad a d>1, \quad b<0 .
\end{aligned}
$$

The whole system of the filter designed by the LCT, i.e., canonical filter system, (17), can be implemented as in Fig. 3. We use $H[\operatorname{sgn}(b) u]$ instead of $H(u)$ as the transfer function and $x_{0}(-t)$ instead of $x_{0}(t)$ as the output, and the parameters of the LCT that we implement depend on

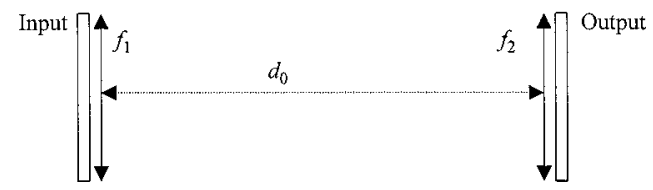

Fig. 1. Implementation of a LCT with two cylinder lenses and one free space.

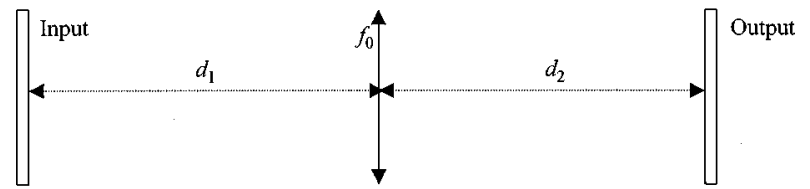

Fig. 2. Implementation of a LCT with one cylinder lens and two free spaces. 

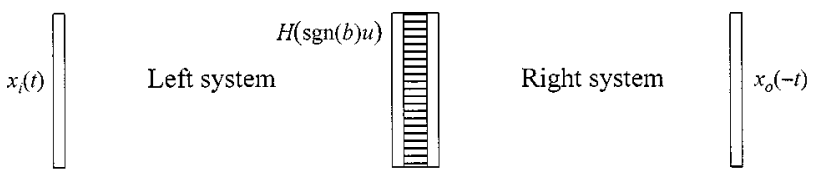

Fig. 3. Optical implementation (OPI) for the filter designed by the LCT. For $b>0$ : Left system, the OPI of the LCT with parameters $\{a, b, c, d\}$; right system, the OPI of the LCT with parameters $\{-d, b, c,-a\}$. For $b<0$ : left system, the OPI of the LCT with parameters $\{-a,-b,-c,-d\}$; right system, the OPI of the LCT with parameters $\{d,-b,-c, a\}$.

the sign of $b$. With these choices, we can ensure that the second parameters of the LCT's for the forward and inverse transforms in Fig. 3 are always positive; and then we can ensure that it is possible to use the method of Fig. 1 to implement both the left- and the right-hand systems. Under some conditions we can also use the method of Fig. 2 to implement the left-hand system or the right-hand system.

Now we discuss the advantages of a type 1 SFRFT for the implementation optical system. The type 1 SFRFT is a special case of a LCT with parameters $\{a, b, c, d\}$ $=\{\cot \alpha, 1,-1,0\}$. Substituting these parameters into Eqs. (38) and (41), we find that, when we use the system shown in Fig. 1 or Fig. 2 to implement the type 1 inverse SFRFT,

for Fig. $1 \quad f_{1}=k(1-\cot a)^{-1}, \quad d_{0}=k, \quad f_{2}=k$;

for Fig. $2 d_{1}=k, \quad f_{0}=k, \quad d_{2}=k(1-\cot \alpha)$.

Note that when we use Fig. 1 or 2 to implement the type 1 SFRFT, although all three components cannot be saved and two of the components are fixed, only the value of $f_{1}$ in Fig. 1 and of $d_{2}$ in Fig. 2 will vary with $\alpha$. So, for the design of a canonical filter when we adjust parameter $\alpha$ to find the optimal filter we need to adjust only one component. This would make the design of a fractional filter convenient, and the original FRFT, the other SFRFT's introduced in this paper, and even the type 2 SFRFT introduced in Subsection 2.B below will not have this advantage. For these transforms, when the values of $\alpha$ is changed, then all the optical components in Figs. 1 and 2 must be adjusted.

For an inverse SFRFT of type 1, i.e., the special case of a LCT for which $\{a, b, c, d\}=\{0,-1,1$, $\cot \alpha\}$, we can implement the LCT with $\{a, b, c, d\}$ $=\{0,1,-1,-\cot \alpha\}$ to avoid a negative length of the free space. By substituting $\{a, b, c, d\}=\{0,1,-1,-\cot \alpha\}$ into Eqs. (38) and (41) we find that when we use Fig. 1 or 2 to implement the inverse SFRFT of type 1

for Fig. $1 f_{1}=k, \quad d_{0}=k, \quad f_{2}=k(1+\cot \alpha)^{-1}$;

for Fig. $2 d_{1}=k(1+\cot \alpha), \quad f_{0}=k, \quad d_{2}=k$.

Thus for the inverse SFRFT of type 1, we again need to adjust only one component for a different value of $\alpha$, and we can implement the fractional filter designed by the type 1 SFRFT as shown in Fig. 4. Here we use the method of Fig. 2 to implement both the forward and that inverse transforms, so the following constraint must be satisfied:

$$
-1<\cot \alpha<1 .
$$

Note from Fig. 4 that, if the width of the filter is ignored, the total length of the fractional filter system is fixed at $8 \pi / \lambda$ (independently of $\alpha$ ). Besides, we need to adjust the lengths of only the center two free spaces for different values of $\alpha$, then the total lengths of the center two free spaces will be fixed at $4 \pi / \lambda$. So when $\alpha$ changes we need to move the location only of filter $H(u)$.

If we use the method of Fig. 1 for the forward and inverse transforms, then the total length is $4 \pi / \lambda$ and is also independent of $\alpha$. When $\alpha$ changes, we need to adjust the focal lengths of only two lenses. Although this method requires adjusting the focal lengths and is not so convenient as the method of Fig. 4 (which requires adjusting only the location of the filter), there is no constraint on $\alpha$. The method of Fig. 4 requires the constraint of inequality (48). Thus, besides its digital implementation, the type 1 SFRFT also has some advantages for optical implementation.

We list some properties of the type 1 SFRFT:

- Convertibility

$$
\begin{aligned}
F_{\beta}(u)=[ & j 2 \pi(\cot \alpha-\cot \beta)]^{-1 / 2} \\
& \times \int_{-\infty}^{\infty} \exp \left[\frac{j(u-t)^{2}}{2(\cot \alpha-\cot \beta)}\right] F_{\alpha}(t) \mathrm{d} t,
\end{aligned}
$$

where

$$
F_{\alpha}(u)=O_{F(1)}^{\alpha}[f(t)], \quad F_{\beta}(u)=O_{F(1)}^{\beta}[f(t)] .
$$

This property comes from the fact that

$$
\left[\begin{array}{cc}
\cot \beta & 1 \\
-1 & 0
\end{array}\right]=\left[\begin{array}{cc}
1 & \cot \alpha-\cot \beta \\
0 & 1
\end{array}\right]\left[\begin{array}{cc}
\cot \alpha & 1 \\
-1 & 0
\end{array}\right] .
$$

Thus, although type 1 SFRFT has no additive properties, it is convertible. We can convert a SFRFT with parameter $\alpha$ into a SFRFT with parameter $\beta$.

- Time shifting property

$$
O_{F(1)}^{\alpha}[f(t-\tau)]=\exp (-j \tau u) F_{\alpha}(u-\tau \cot \alpha) .
$$

- Modulation property:

$$
O_{F(1)}^{\alpha}[\exp (j v t) f(t)]=F_{\alpha}(u-v),
$$

as for the original Fourier transform.

- Differentiation property

$$
O_{F(1)}^{\alpha}\left[f^{\prime}(t)\right]=\cot \alpha F_{\alpha}^{\prime}(u)+j u F_{\alpha}(u) .
$$

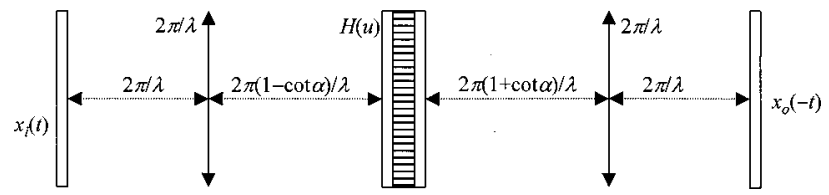

Fig. 4. Optical implementation of the fractional filter designed by the type 1 SFRFT. 
- Multiplication property:

$$
O_{F(1)}^{\alpha}[-j t f(t)]=F_{\alpha}^{\prime}(u),
$$

as for the original Fourier transform.

- Divisibility

$$
O_{F(1)}^{\alpha}[f(t) / t]=-j \int_{-\infty}^{u} F_{\alpha}(p) \mathrm{d} p
$$

as for the original Fourier transform.

In summary, the SFRFT of type 1 has the following advantages:

(1) The complexity of digital implementation is reduced from $2 P+(P / 2) \log _{2} P$ to $P+(P / 2) \log _{2} P$.

(2) For the digital implementation algorithm introduced in Ref. 9 (which requires only one fast Fourier transform for implementing the FRFT or the LCT), the product of $\Delta_{t} \Delta_{u}$ is fixed to $2 \pi /(2 N+1)$ and does not vary with $\alpha$.

(3) The fractional convolution requires only one integration operation.

(4) For optical implementation of the fractional filter, the total length is fixed for all $\alpha$.

(5) For optical implementation of the fractional filter, when $\alpha$ changes we need to adjust only two optical components.

(6) Some properties of the type 1 SFRFT, such as the modulation, multiplication, and division properties, are the same as those of the original Fourier transform. The corresponding properties of the FRFT are different from those of the original Fourier transform.

\section{Type 2 SFRFT}

The type 1 SFRFT is suitable for both digital and optical implementation. When we use the optical system, the size of the system is fixed for all $\alpha$, and, when $\alpha$ changes, we need to adjust only two of the optical components. But we still need three optical components for the type 1 SFRFT, and for the whole system of the fractional filter we still need six. In this subsection we introduce the type 2 SFRFT. We need only two optical components to implement it. Although implementation of its optical system is not of a fixed size and all the components must be adjusted when $\alpha$ is changed, if the number of optical components is the major consideration, the type 2 SFRFT will be well suited for optical implementation.

For the type 2 SFRFT we choose

$$
a=1, \quad d=-1, \quad a / b=\cot \alpha,
$$

and the parameters of the LCT become $\{a, b, c, d\}$ $=\{1, \tan \alpha,-2 \cot \alpha,-1\}$. Then we obtain the formula for a type 2 SFRFT:

\section{Type 2 SFRFT}

$$
\begin{aligned}
O_{F(2)}^{\alpha}[f(t)]= & \left(\frac{\cot \alpha}{j 2 \pi}\right)^{1 / 2} \exp \left(-\frac{j}{2} u^{2} \cot \alpha\right) \\
& \times \int_{-\infty}^{\infty} \exp \left(-j u t \cot \alpha+\frac{j}{2} t^{2} \cot \alpha\right) \\
& \times f(t) \mathrm{d} t .
\end{aligned}
$$

From the fact that the inverse of the LCT with parameters $\{a, b, c, d\}$ is the LCT with parameters $\{d,-b$, $-c, a\}$, so the inverse type $2 \mathrm{SFRFT}$ is the LCT with parameters $\{-1,-\tan \alpha, 2 \cot \alpha, 1\}$. That is,

$$
\begin{aligned}
O_{I F(2)}^{\alpha}\left[F_{\alpha}(u)\right]= & \left(\frac{j \cot \alpha}{2 \pi}\right)^{1 / 2} \exp \left(-\frac{j}{2} t^{2} \cot \alpha\right) \\
& \times \int_{-\infty}^{\infty} \exp \left(j u t \cot \alpha+\frac{j}{2} u^{2} \cot \alpha\right) \\
& \times F_{\alpha}(u) \mathrm{d} t
\end{aligned}
$$

The SFRFT is similar to the original FRFT, except that the outside chirp term $\exp \left(j u^{2} \cot \alpha / 2\right)$ is changed into $\exp \left(-j u^{2} \cot \alpha / 2\right)$.

The type 2 SFRFT will have the advantage of simpler optical implementation. From Eqs. (38) and (41) we note that when $a=1$ or $d=1$, then in Fig. 1 one of the lenses will have an infinite focal length and can be removed, and in Fig. 2 one of the free spaces will have zero length. So, when we use either Fig. 1 or Fig. 2 to implement the LCT, if we set $a=1$ or $d=1$ we can save one optical component. Then we consider the optical implementation of the fractional filter as shown in Fig. 3. If we set $a=1$ and $d=-1$, for both $b>0$ and $b<0$ we can save one optical component in the left-hand system, and one in the in the right-hand system, for a total saving of two optical components. This is why we chose $a=1$ and $d=-1$ in Eq. (55).

When we substitute $\{a, b, c, d\}=\{1, \tan \alpha,-2 \cot \alpha$, $-1\}$ into Eqs. (38) and (41), we obtain

$$
\begin{aligned}
& \text { for Fig. } 1 \quad f_{1} \rightarrow \infty, \quad d_{0}=k \tan \alpha, \\
& f_{2}=(k / 2) \tan \alpha ; \\
& \text { for Fig. } 2 \quad d_{1}=k \tan \alpha, \quad f_{0}=(k / 2) \tan \alpha, \\
& d_{2}=0 .
\end{aligned}
$$

That is, when we use the methods of Figs. 1 and 2 to implement the type 2 SFRFT, for Fig. 1 the first lens can be removed and for Fig. 2 the second free space can be removed. In as much as $d_{0}=d_{1}$ and $f_{2}=f_{0}$, for a type 2 SFRFT optical implementation by the method of Fig. 1 is the same as optical implementation by the method of Fig. 2, as we illustrate in Fig. 5. Note from Fig. 5 that the length of free space is always twice the focal length of the lens, no matter what the value of $\alpha$ is. The configuration of Fig. 5 is suitable when $\tan \alpha>0$. When $\tan \alpha<0$, we can implement the LCT with parameters $\{-a,-b,-c,-d\}=\{-1,-\tan \alpha, 2 \cot \alpha, 1\}$ instead of $\{1, \tan \alpha,-2 \cot \alpha,-1\}$.

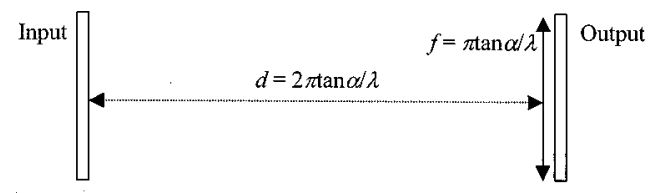

Fig. 5. Optical implementation of the type 2 SFRFT $(\tan \alpha$ $>0)$. 


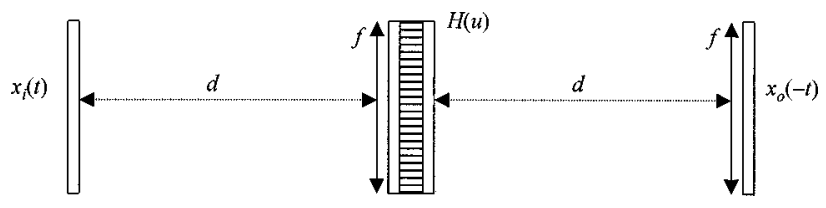

Fig. 6. Filter designed by the type $2 \operatorname{SFRFT}(\tan \alpha>0)$.

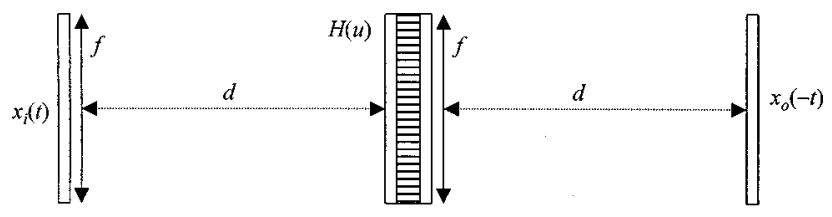

Fig. 7. Filter designed by the type 2 SFRFT when $\tan \alpha<0$.

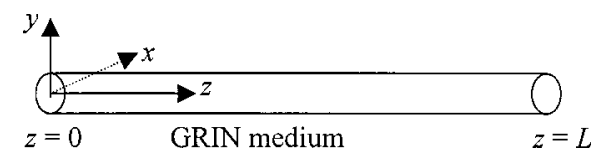

Fig. 8. GRIN medium and directions of the $x, y$, and $z$ axes.

For the fractional filter system designed by a type 2 SFRFT we can use the system based on Fig. 3 and implement the whole system as illustrated in Fig. 6 when $\tan \alpha>0$, where

$$
d=k \tan \alpha, \quad f=(k / 2) \tan \alpha .
$$

When $\tan \alpha<0$ we can use the implementation of Fig. 7 and the values of $f$ and $d$ will be

$$
f=(k / 2)|\tan \alpha|, \quad d=k|\tan \alpha| .
$$

In that in both Figs. 6 and 7 the inverse system is the same as the forward system. So the two advantages of using a type 2 SFRFT are that we need only two lenses, two free spaces, and therefore a total of four optical components for a fractional filter and that the forward and the inverse systems have the same structure.

\section{Type 3 SFRFT}

The type 3 SFRFT is suitable for implementation of a GRIN medium. Suppose that a GRIN medium has the following refractive-index distribution:

$$
\begin{array}{r}
n^{2}(x, y)=n_{0}^{2}\left[1-\left(n_{x} / n_{0}\right) x^{2}-\left(n_{y} / n_{0}\right) y^{2}\right], \\
n_{x}, n_{y} \ll n_{0} .
\end{array}
$$

And suppose that in Fig. 8 the field distribution at $z$ $=0$ is $f_{0}(x, y)$ and the field distribution at $z=L$ is $f_{L}(x, y)$. Then the relationship between $f_{0}(x, y)$ and $f_{L}(x, y)$ in Fig. 8 is ${ }^{18}$

$$
\begin{aligned}
f_{L}(x, y)= & \exp \left(-j \frac{2 \pi n_{0} L}{\lambda}\right) O_{F x}^{\left(a_{x}, b_{x}, c_{x}, d_{x}\right)} \\
& \times\left\{O_{F y}^{\left(a_{y}, b_{y}, c_{y}, d_{y}\right)}\left[f_{0}(x, y)\right]\right\} .
\end{aligned}
$$

Here we use $O_{F x}$ and $O_{F y}$ to denote the one-dimensional LCT along the $x$ axis and the $y$ axis, and

$$
\left[\begin{array}{ll}
a_{x} & b_{x} \\
c_{x} & d_{x}
\end{array}\right]=\left[\begin{array}{cc}
\cos \phi & w_{x} \sin \phi \\
-\frac{\sin \phi}{w_{x}} & \cos \phi
\end{array}\right],
$$

$$
\left[\begin{array}{ll}
a_{y} & b_{y} \\
c_{y} & d_{y}
\end{array}\right]=\left[\begin{array}{cc}
\cos \varphi & w_{y} \sin \varphi \\
-\frac{\sin \varphi}{w_{y}} & \cos \varphi
\end{array}\right],
$$

where

$$
\begin{aligned}
w_{x} & =(1 / k)\left(n_{x} n_{0}\right)^{-1 / 2}, \quad w_{y}=(1 / k)\left(n_{y} n_{0}\right)^{-1 / 2}, \\
\phi & =L\left(n_{x} / n_{0}\right)^{1 / 2}, \quad \varphi=L\left(n_{y} / n_{0}\right)^{1 / 2} .
\end{aligned}
$$

So the effect of the GRIN medium is similar to that of a FRFT, except that here $\sin \alpha$ is converted into $w_{x} \sin \alpha$ and $-\sin \alpha$ is converted into $-\sin \alpha / w_{x}$. It seems that we can define the new type of FRFT according to the following conversions:

$$
\begin{array}{r}
O_{F(3)}^{\phi}[f(t)]=O_{F}^{\left(\cos \phi, w_{x} \sin \phi,-\sin \phi / w_{x}, \cos \phi\right)}[f(t)], \\
\phi \geqslant 0, \quad \text { i.e., } L \geqslant 0 .
\end{array}
$$

Type 3 SFRFT: $(\phi \geqslant 0)$

So the definition of the type 3 SFRFT is

$$
\begin{aligned}
O_{F(3)}^{\phi}[f(t)]= & \left(\frac{\csc \phi}{j 2 \pi w_{x}}\right)^{1 / 2} \exp \left(\frac{j}{2 w_{x}} u^{2} \cot \phi\right) \\
& \times \int_{-\infty}^{\infty} \exp \left(-j u t \frac{\csc \phi}{w_{x}}+\frac{j}{2 w_{x}} t^{2} \cot \phi\right) \\
& \times f(t) \mathrm{d} t .
\end{aligned}
$$

We call Eq. (68) a type 3 SFRFT. Although the type 3 SFRFT seems very similar to the original FRFT, we have shown that for filter design the ratio $b / a$ is important and that for the type $3 \mathrm{FRFT}$

$$
b / a=w_{x} \tan \phi .
$$

So the type 3 FRFT with order $\phi$ is different from the original FRFT of order $\alpha$ for design of fractional filters. Instead, it is equivalent to the original FRFT with order $\alpha$, and the relationship between $\phi$ and $\alpha$ is

$$
\alpha=\tan ^{-1}\left(w_{x} \tan \phi\right) \quad \text { or } \quad \phi=\tan ^{-1}\left(w_{x}^{-1} \tan \alpha\right) .
$$

So, from Eqs. (67) and (70), if we want the GRIN medium to act as a FRFT of order $\alpha$ in the $x$ axis, then the length of the GRIN medium should be

$$
L=\left(n_{0} / n_{x}\right)^{1 / 2} \tan ^{-1}\left(w_{x}^{-1} \tan \alpha\right) .
$$

Now we discuss the inverse of the type 3 SFRFT. Inasmuch as

$$
\begin{aligned}
{\left[\begin{array}{cc}
\cos \phi & w_{x} \sin \phi \\
-w_{x}^{-1} \sin \phi & \cos \phi
\end{array}\right]^{-1} } & \\
& =\left[\begin{array}{cc}
\cos \phi & -w_{x} \sin \phi \\
w_{x}^{-1} \sin \phi & \cos \phi
\end{array}\right] \\
& =\left[\begin{array}{cc}
\cos (-\phi) & w_{x} \sin (-\phi) \\
-w_{x}^{-1} \sin (-\phi) & \cos (-\phi)
\end{array}\right],
\end{aligned}
$$

it would seem that the inverse of the type 3 SFRFT with parameter $\phi$ would be the type 3 SFRFT with parameter $-\phi$. But, because length $L$ of the GRIN medium should be nonnegative and in Eq. (67) $L$ is negative when $\phi$ 
$<0$, we use $2 \pi-\phi$ instead of $\phi$. So the inverse of the type 3 SFRFT with parameter $\phi$ is the type 3 SFRFT with parameter $2 \pi-\phi(\phi>0)$ :

$$
O_{F(3)}^{2 \pi-\phi}\left\{O_{F(3)}^{\phi}[f(t)]\right\}=f(t) .
$$

The type 3 SFRFT will have the additive, periodic properties

$$
\begin{gathered}
O_{F(3)}^{\phi}\left\{O_{F(3)}^{\varphi}[f(t)]\right\}=O_{F(3)}^{\varphi}\left\{O_{F(3)}^{\phi}[f(t)]\right\}=O_{F(3)}^{\phi+\varphi}[f(t)], \\
O_{F(3)}^{\phi}[f(t)]=O_{F(3)}^{\phi+2 \pi}[f(t)] .
\end{gathered}
$$

We note that, although other SFRFTs' introduced in this paper will have no additive and periodic properties, these properties will exist for the type $3 \mathrm{SFRFT}$. This is the advantage of the type 3 SFRFT and hence is the advantage of using the GRIN medium to implement the fractional filter. Because of these properties, when we use the GRIN medium to design the fractional filter the total length of the system is independent of the value of $\phi$.

The fractional filter system design by the type 3 SFRFT can be written as

$$
x_{0}(t)=O_{F(3)}^{2 \pi-\phi}\left\{O_{F(3)}^{\phi}\left[x_{i}(t)\right] H(u)\right\},
$$

or

$$
x_{0}(-t)=O_{F(3)}^{\pi-\phi}\left\{O_{F(3)}^{\phi}\left[x_{i}(t)\right] H(u)\right\}, \quad \phi<\pi,
$$

and it can be implemented by the GRIN medium as shown in Fig. 9, where the values of $L_{1}$ and $L_{2}$ are

$$
L_{1}=\phi\left(n_{0} / n_{x}\right)^{1 / 2}, \quad L_{2}=(\pi-\phi)\left(n_{0} / n_{x}\right)^{1 / 2} .
$$

We use $x_{0}(-t)$ instead of $x_{0}(t)$ as the output to reduce the length of the GRIN medium. Because, from Eq. (67), length $L$ of the GRIN medium is proportional to order $\phi$, and, if we use $x_{0}(t)$ as the output, we must use the type 3 SFRFT with order $2 \pi-\phi$ for the inverse transform and a larger value of $L_{2}$ will be required. We also note that, if the thickness of filter $H(u)$ is not considered, the total length of the filter system in Fig. 9 will be $L_{1}+L_{2}$ $=\pi\left(n_{0} / n_{x}\right)^{1 / 2}$ and will always be independent of $\phi$. The fixed size of the implementation is the advantage that we derive using the GRIN medium to implement the fractional filter.

For the fractional filter of Fig. 9 the value of $\phi$ is constrained in the range $(0<\phi<\pi)$. This seems to be a restriction. But, for the type $3 \mathrm{SFRFT}, \cot \alpha=w_{x} \tan \phi$ ( $\alpha$ is of the order of the FRFT), so we can obtain all the values of $\alpha$ by varying $\phi$ just in the range $(0<\phi<\pi)$. So we can still use the type 3 SFRFT to implement the fractional filter for any value of $\alpha$.

\section{E. Type 4 SFRFT}

The type 4 SFRFT is suitable when we use the radar system to implement the FRFT. The relationship between the monochromic light propagating between the spherical

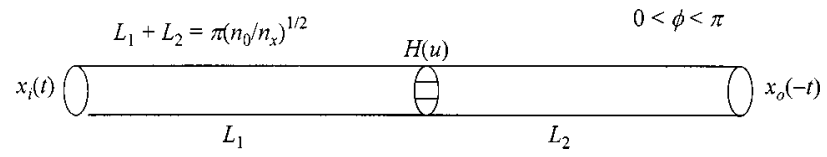

Fig. 9. GRIN medium implementation of the fractional filter designed by the type 3 SFRFT.

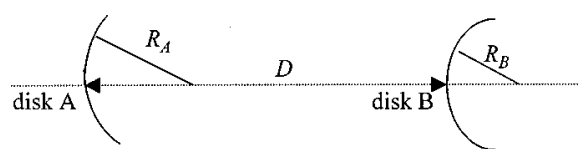

Fig. 10. This system contains two spherical disks and one free space.

emitters and receivers and the LCT was discussed in detail in Ref. 19. Suppose that there are two spherical disks, $\mathrm{A}$ and $\mathrm{B}$, as in Fig. 10, where $R_{A}$ and $R_{B}$ are the radii of the spherical disks $\mathrm{A}$ and $\mathrm{B}$, respectively, and $D$ is the distance between the vertices of disks $\mathrm{A}$ and $\mathrm{B}$. If the field distribution on disk $\mathrm{A}$ is $F_{A}(x, y)$ and the field distribution on disk $\mathrm{B}$ is $F_{B}(s, h)$, then, as was discussed in Ref. 19, the relationship between $F_{A}(x, y)$ and $F_{B}(s, h)$ :

$$
\begin{aligned}
F_{B}(k, h)= & \exp \left(-j 2 \pi D \lambda^{-1}\right) O_{S x}^{\left(R_{A}, R_{B}, D\right)} \\
& \times\left\{O_{S y}^{\left(R_{A}, R_{B}, D\right)}\left[F_{A}(x, y)\right]\right\},
\end{aligned}
$$

where the one-dimensional transform $O_{S x}^{\left(R_{A}, R_{B}, D\right)}$ is

$$
\begin{aligned}
O_{S x}^{\left(R_{A}, R_{B}, D\right)}[f(x)] & \sqrt{\frac{j}{\lambda D}} \exp \left[-\frac{j \pi}{\lambda}\left(R_{B}^{-1}+D^{-1}\right) s^{2}\right] \int_{-\infty}^{\infty} \exp \left[\frac{j 2 \pi}{\lambda D} s x\right. \\
= & \left.\frac{j \pi}{\lambda}\left(R_{A}^{-1}-D^{-1}\right) s^{2}\right] f(x) \mathrm{d} x, \quad D \neq 0, \quad(79) \\
& +{ }^{\left(R_{A}, R_{B}, 0\right)}[f(x)] \\
= & \exp \left[\frac{j \pi}{\lambda}\left(R_{A}^{-1}-R_{B}^{-1}\right) s^{2}\right] f(s), \quad D=0,
\end{aligned}
$$

and $O_{S y}^{\left(R_{A}, R_{B}, D\right)}[f(y)]$ is of the same form as $O_{S x}^{\left(R_{A}, R_{B}, D\right)}$ $\times[f(x)]$, except that the variables $\{x, k\}$ are changed into $\{y, h\}$. We note that $O_{S x}^{\left(R_{A}, R_{B}, d\right)}$ is just the LCT with the parameters

$$
\left[\begin{array}{ll}
a & b \\
c & d
\end{array}\right]=\left[\begin{array}{cc}
1-R_{A}^{-1} D & -D / k \\
k\left(R_{A}^{-1}-R_{B}^{-1}+R_{A}^{-1} R_{B}^{-1} D\right) & 1+R_{B}^{-1} D
\end{array}\right] .
$$

So we can use the radar system to implement the LCT, and, as length $D$ should be nonnegative, we can use the radar system to implement the LCT when

$$
b \leqslant 0 .
$$

We already know that the ratio $b / a$ will affect the properties of the fractional filter designed by the LCT. In Eq. (81),

$$
\frac{b}{a}=k^{-1}\left(R_{A}^{-1}-D^{-1}\right)^{-1} .
$$

We can fix the value of $R_{A}$ and adjust length $D$ of the free space according Eq. (84) as below to obtain the desired value of $b / a$. Here we decide to set the relationship between $R_{A}$ and $D$ as

$$
R_{A}=D / 2
$$


to make the value of $a$ be -1 . Also, the value of $R_{B}$ will have no effect on the fractional filter, so we can set $R_{B}$ to be any value. We choose

$$
R_{B} \rightarrow \infty .
$$

After substituting Eq. (84) and relation (85) into Eq. (81), we obtain

$$
\left[\begin{array}{ll}
a & b \\
c & d
\end{array}\right]=\left[\begin{array}{cc}
-1 & -D / k \\
2 k / D & 1
\end{array}\right],
$$

so we can define the type 4 SFRFT as

\section{Type 4 SFRFT}

$$
\begin{aligned}
O_{F(4)}^{(D)}[f(x)]= & \sqrt{\frac{j}{\lambda D}} \exp \left(-\frac{j k}{2 D} s^{2}\right) \\
& \times \int_{-\infty}^{\infty} \exp \left(\frac{j k}{D} s x+\frac{j k}{2 D} x^{2}\right) f(x) \mathrm{d} x .
\end{aligned}
$$

Since $b / a=D / k$, we can use the value of $D$ to control the effects of the type 4 SFRFT for the design of the fractional filter. To make the type 4 SFRFT have the same effects as the original FRFT of order $\alpha$ for the design of the fractional filter we can set

$$
D=k \tan \alpha .
$$

Then we discuss a fractional filter designed by the type 4 SFRFT. Inasmuch as

$$
f(-x)=O_{F(4)}^{(D)}\left\{O_{F(4)}^{(D)}[f(x)]\right\},
$$

the inverse of the type 4 SFRFT with parameter $D$ is just the forward type 4 SFRFT with parameter $D$ itself. And the formula for the fractional filter system designed by the type 4 SFRFT is

$$
f_{0}(-x)=O_{F(4)}^{(D)}\left\{O_{F(4)}^{(D)}\left(f_{i}(x)\right) H(s)\right\} .
$$

It can be implemented as in Fig. 11. Note from Fig. 11 that there are only two spherical disks with radii that vary with $D$ and hence vary with $\alpha$. The other two spherical disks have infinite radii and can be replaced by plane receivers, which would lessen the cost of the implementation. When the radar system is used to implement the fractional filter designed by the original FRFT, the four spherical disks will all have finite radii and can not be replaced by plane emitters or receivers, and the structures of the forward and inverse transform systems will be different. So using the type 4 SFRFT will indeed make the implementation of the fractional filter by the radar system much simpler.

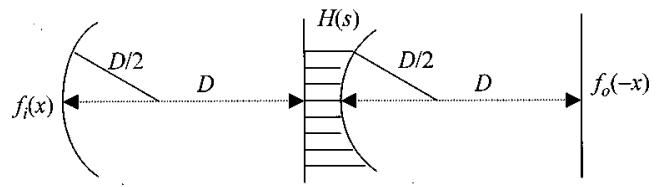

Fig. 11. Radar system to implement the fractional filter (designed by type 4 SFRFT).

\section{SFRFT FOR FRACTIONAL CORRELATION}

In Section 2 we discussed the conditions under which the LCT will have the same effects as the FRFT with order $\alpha$ for filter design, and we used the result to simplify the FRFT for digital computation and optical, GRIN-medium, and radar-system implementation. In this section we discuss under what conditions the LCT will have the same effects as the FRFT with order $\alpha$ for fractional correlation and use these results to define other types of the simplified FRFT.

A. Canonical Correlation and Effects of the Parameters Fractional correlation ${ }^{20,21}$ is defined as

$$
O_{\text {corr }}^{p, q, r}[x(t), y(t)]=O_{F}^{r}\left\{O_{F}^{p}[x(t)] \overline{O_{F}^{q}[y(t)]}\right\},
$$

where the overbar means complex conjugation and

$$
\cot p+\cot r=\cot q .
$$

It has been used for space-variant pattern recognition. ${ }^{21}$ It can not only find the objects that are the same as the reference pattern but also predict whether these objects will be in a certain region. In Ref. 22 the use of fractional correlation for scaled pattern recognition, that is, detecting the objects that have the same scale as the reference, is discussed.

We can further generalize the fractional correlation into a canonical correlation by replacing the FRFT's with LCT's:

$$
\begin{aligned}
& O_{\text {corr }}^{(a, b, c, d),(e, f, g, h)(m, n, s, v)}[x(t), y(t)] \\
& =O_{F}^{(m, n, s, v)}\left\{O_{F}^{(a, b, c, d)}[x(t)] \overline{O_{F}^{(e, f, g, h)}[y(t)]}\right\} .
\end{aligned}
$$

Because we have not seen the canonical correlation discussed in easier papers, we discuss it in detail here. If we use $z(t)$ to denote the output of the canonical correlation of $x(t)$ and $y(t)$, then

$$
\begin{aligned}
z(t)= & \frac{1}{2 \pi}\left(\frac{j}{2 \pi b f n}\right)^{1 / 2} \exp \left(\frac{j v}{2 n} t^{2}\right) \\
& \times \int_{-\infty}^{\infty} \exp \left(-\frac{j}{n} u t+\frac{j m}{2 n} u^{2}\right) \exp \left(\frac{j a}{2 b} u^{2}\right) \\
& \times \int_{-\infty}^{\infty} \exp \left(-\frac{j}{b} u t+\frac{j a}{2 b} u^{2}\right) x(\tau) \mathrm{d} \tau \exp \left(-\frac{j k}{2 f} u^{2}\right) \\
& \times \int_{-\infty}^{\infty} \exp \left(\frac{j}{f} u \eta-\frac{j e}{2 f} \eta^{2}\right) \overline{y(n)} \mathrm{d} \eta \mathrm{d} u \\
= & \frac{1}{2 \pi}\left(\frac{j}{2 \pi b f n}\right)^{1 / 2} \exp \left(\frac{j v}{2 n} t^{2}\right) \\
& \times \int_{-\infty}^{\infty} \int_{-\infty}^{\infty} \exp \left(\frac{j a}{2 b} \tau^{2}-\frac{j e}{2 f} \eta^{2}\right) \\
& \times \int_{-\infty}^{\infty} \exp \left[j u\left(-\frac{t}{n}-\frac{\tau}{b}+\frac{\eta}{f}\right)\right] \\
& \times \exp \left[\frac{j}{2}\left(\frac{n}{m}+\frac{d}{b}-\frac{h}{f}\right) u^{2}\right] \mathrm{d} u x(\tau) \overline{y(\eta)} \mathrm{d} \tau \mathrm{d} \eta .
\end{aligned}
$$


We require that

$$
\frac{d}{b}+\frac{m}{n}=\frac{h}{f}
$$

Then

$$
\begin{aligned}
& z(t)=\left(\frac{j}{2 \pi b f n}\right)^{1 / 2} \exp \left(\frac{j v}{2 n} t^{2}\right) \\
& \times \int_{-\infty}^{\infty} \int_{-\infty}^{\infty} \exp \left(\frac{j a}{2 b} \tau^{2}-\frac{j e}{2 f} \eta^{2}\right) \\
& \times \delta\left(-\frac{t}{n}-\frac{\tau}{b}+\frac{\eta}{f}\right) x(\tau) \overline{y(\eta)} \mathrm{d} \tau \mathrm{d} \eta \\
& =\left(\frac{j f}{2 \pi b n}\right)^{1 / 2} \exp \left(\frac{j v}{2 n} t^{2}\right) \\
& \times \int_{-\infty}^{\infty} \exp \left[\frac{j a}{2 b} \tau^{2}-\frac{j e f}{2}\left(\frac{t}{n}+\frac{\tau}{b}\right)^{2}\right] \\
& \times x(\tau) y *\left(\frac{f t}{n}+\frac{f \tau}{b}\right) \mathrm{d} \tau \\
& =\left(\frac{j f}{2 \pi b n}\right)^{1 / 2} \exp \left[\frac{j(v n-e f)}{2 n^{2}} t^{2}\right] \\
& \times \int_{-\infty}^{\infty} \exp \left[\frac{j(a b-e f)}{2 b^{2}} \tau^{2}-j \frac{e f}{n b} t \tau\right] \\
& \times x(\tau) y *\left(\frac{f t}{n}+\frac{f \tau}{b}\right) \mathrm{d} \tau, \\
& |z(t)|=\left(\frac{|f|}{2 \pi|b n|}\right)^{1 / 2} \mid \int_{-\infty}^{\infty} \exp \left[\frac{j(a b-e f)}{2 b^{2}} \tau^{2}\right. \\
& \left.-j \frac{e f}{n b} t \tau\right] x(\tau) y^{*}\left(\frac{f t}{n}+\frac{f \tau}{b}\right) \mathrm{d} \tau \mid .
\end{aligned}
$$

Then suppose that $y(t)$ is the scaling of $x(t)$ with displacement $t_{0}$ :

$$
y(t)=x\left[\frac{b}{f}\left(t-t_{0}\right)\right] .
$$

Then

$$
\begin{aligned}
|z(t)|= & \left(\frac{|f|}{2 \pi|b n|}\right)^{1 / 2} \mid \int_{-\infty}^{\infty} \exp \left[\frac{j(a b-e f)}{2 b^{2}} \tau^{2}\right. \\
& \left.-j \frac{e f}{n b} t \tau\right] x(\tau) x^{*}\left(\tau+\frac{b}{n} t-\frac{b}{f} t_{0}\right) \mathrm{d} \tau \mid, \\
\left|z\left(\frac{n}{f} t_{0}\right)\right|= & \left(\frac{|f|}{2 \pi|b n|}\right)^{1 / 2} \\
& \times \mid \int_{-\infty}^{\infty} \exp \left[\frac{j(a b-e f)}{2 b^{2}} \tau^{2}-j \frac{e}{b} t_{0} \tau\right] \\
& \times|x(\tau)|^{2} \mathrm{~d} \tau \mid .
\end{aligned}
$$

When $a b=e f$,

$$
\left|z\left(\frac{n}{f} t_{0}\right)\right|=\left.\left(\frac{|f|}{2 \pi|b n|}\right)^{1 / 2}\left|\int_{-\infty}^{\infty} \exp \left(-j \frac{e}{b} t_{0} \tau\right)\right| x(\tau)\right|^{2} \mathrm{~d} \tau \mid ;
$$

and when $a b \neq e f$,

$$
\begin{aligned}
\left|z\left(\frac{n}{f} t_{0}\right)\right|= & \left.\frac{|f|}{2 \pi|b n|}\right)^{1 / 2} \mid \int_{-\infty}^{\infty} \exp \left[\frac{j e^{2}}{2(a b-e f)}\right. \\
& \left.\times\left(t_{0}-\frac{a b-e f}{b e} \tau\right)^{2}\right]|x(\tau)|^{2} \mathrm{~d} \tau \mid .
\end{aligned}
$$

If we want $\left|z\left(n t_{0} / f\right)\right|$ [i.e., the peak of $|z(t)|$ ] to be sufficiently large, the phase of the exponential term should be restricted in the range $[-\pi, \pi]$. Thus when

$$
a b=e f,
$$

if $y(t)$ is the scaling of $x(t)$ with the factor $f / b$ with some displacement, i.e., $y(t)=x\left[b\left(t-t_{0}\right) / f\right]$, and displacement $t_{0}$ is in the range

$$
-\left|\begin{array}{ll}
\frac{\pi}{B} & \frac{b}{e}
\end{array}\right|<t_{0}<\left|\frac{\pi}{\bar{B}} \frac{b}{e}\right|
$$

where $B$ is defined as

$$
x(t) \approx 0, \quad|t|>B,
$$

then $|z(t)|$ will have its peak at $t=n t_{0} / f$ with no serious distortion. But, when

$$
a b \neq e f,
$$

if $y(t)$ is the scaling of $x(t)$ with the factor $f / b$ with some displacement, i.e., $y(t)=x\left[b\left(t-t_{0}\right) / f\right]$, and the displacement $t_{0}$ is in the range

$$
\begin{aligned}
\left|\frac{a}{e}-\frac{f}{b}\right| B & -\left[2 \pi\left|\frac{b}{e}\left(\frac{a}{e}-\frac{f}{b}\right)\right|\right]^{1 / 2} \\
& \left.<t_{0}<|2 \pi| \frac{b}{e}\left(\frac{a}{e}-\frac{f}{b}\right) \mid\right]^{1 / 2}-\left|\frac{a}{e}-\frac{f}{b}\right| B,
\end{aligned}
$$

then $|z(t)|$ will have its peak at $t=n t_{0} / f$ with no serious distortion.

In other cases, the peak of $|z(t)|$ will have serious distortion. So, if we choose the parameters properly, we can detect the objects that scale with the reference with a certain scaling factor and are in a certain region. So we can use the canonical correlation for scaled shift-variant pattern recognition.

\section{B. Type 5 SFRFT}

When one uses canonical correlation for pattern recognition, there are only two terms to be decided: the scaling ratio and the width of the region over which the object can be detected. But, for fractional correlation as in Eq. (92), there are a total of 12 parameters. It seems unnecessary to use so many parameters to control only two terms, so we can simplify the canonical correlation by reducing the number of parameters. We can set 
- $\{m, n, s, v\}=\{0,1,-1,0\}$. That is, the last LCT in Eq. (92) becomes the Fourier transform.

- $a=e=1$.

- $d=h=0$. Then, as $m=d=h=0$, the constraint of Eq. (94) will always be satisfied; and, as ad $-b c=e h-f g=1, c=-1 / b$ and $g=-1 / f$.

Now the number of parameters of canonical correlation is reduced from 12 to 2, and Eq. (92) can be rewritten as

$$
\begin{aligned}
O_{\mathrm{corr}}^{b, f}[x(t), y(t)]= & \mathrm{FT}\left\{O_{F}^{\left(1, b,-b^{-1}, 0\right)}[x(t)]\right. \\
& \left.\times \overline{O_{F}^{\left(1, f,-f^{-1}, 0\right)}[y(t)]}\right\} .
\end{aligned}
$$

Equation (97) remains the same, and Eq. (99) is simplified as

$$
\begin{aligned}
\left|z\left(f^{-1} t_{0}\right)\right|= & \left(\frac{|f|}{2 \pi|b|}\right)^{1 / 2} \mid \int_{-\infty}^{\infty} \exp \left[\frac{j(b-f)}{2 b^{2}} \tau^{2}-\frac{j}{b} t_{0} \tau\right] \\
& \times|x(\tau)|^{2} \mathrm{~d} \tau \mid .
\end{aligned}
$$

And the conditions under which we can detect the objects are the following: When

$$
b=f,
$$

if $y(t)$ is the displacement of $x(t)$, i.e.,

$$
y(t)=x\left(t-t_{0}\right),
$$

then displacement $t_{0}$ is in the range

$$
-\left|\frac{b \pi}{B}\right|<t_{0}<\left|\frac{b \pi}{B}\right| .
$$

When

$$
b \neq f,
$$

if $y(t)$ is the scaling of $x(t)$ with the factor $f / b$ with some displacement, i.e.,

$$
y(t)=x\left[b\left(t-t_{0}\right) / f\right],
$$

and displacement $t_{0}$ is in the range

$$
\begin{aligned}
& \left|1-\frac{f}{b}\right| B-\left[2 \pi\left|b\left(1-\frac{f}{b}\right)\right|\right]^{1 / 2}<t_{0} \\
& <\left[2 \pi\left|b\left(1-\frac{f}{b}\right)\right|\right]^{1 / 2}-\left|1-\frac{f}{b}\right| B,
\end{aligned}
$$

then $|z(t)|$ will have its peak at $t=t_{0} / f$ without serious distortion.

The simplified canonical correlation has only two parameters $b$ and $f$, and we can use these two parameters to control the two terms (the scaling ratio and the width of the detecting region). This is much simpler than the original canonical correlation and the original fractional correlation. We can choose the parameters according to the following model:

First, decide the scaling ratio $\sigma[\sigma=$ (width of the object)/(width of the reference)] and the width of detecting region $T$ (which means that the object can be detected when $-T / 2<t_{0}<T / 2$, where $t_{0}$ is the amount of displacement).
Then, because $f / b=\sigma$, and from inequalities (108) and (110), when $\sigma=1$ we can choose $b$ as

$$
b=T B / 2 \pi,
$$

and when $\sigma \neq 1$ we can choose $b$ as

$$
b=\frac{1}{2 \pi(1-\sigma)}\left(\frac{T}{2}+|1-\sigma| B\right)^{2} .
$$

Then we choose $f$ as

$$
f=b \sigma .
$$

The process of deciding the parameters of the simplified canonical correlation is much simpler than the original canonical correlation. When we want to change the width of the detection range or the scaling ratio, we can first adjust $b$ according to Eq. (111) or (112) and then adjust $f$ according to Eq. (113).

From the discussion above, we can define the SFRFT that is suitable for the fractional correlation. We can define the type 5 SFRFT as

\section{Type 5 SFRFT}

$$
\begin{aligned}
F_{b}(u) & =O_{F(5)}^{b}[f(t)] \\
& =(j 2 \pi b)^{-1 / 2} \int_{-\infty}^{\infty} \exp \left(-\frac{j}{b} u t+\frac{j}{2 b} t^{2}\right) f(t) \mathrm{d} t,
\end{aligned}
$$

which corresponds to a LCT with the parameters $\left\{1, b,-b^{-1}, 0\right\}$, and its inverse is the LCT with the parameters $\left\{0,-b, b^{-1}, 1\right\}$ :

$$
\begin{aligned}
f(t) & =O_{I F(5)}^{b}\left[F_{b}(u)\right] \\
& =\left(\frac{j}{2 \pi b}\right)^{1 / 2} \exp \left(-\frac{j}{2 b} t^{2}\right) \int_{-\infty}^{\infty} \exp \left(\frac{j}{b} t u\right) F_{b}(u) \mathrm{d} u .
\end{aligned}
$$

From the definition of the type 5 SFRFT we can rewrite Eq. (92) and define the fractional correlation as

$$
O_{\text {corr }}^{b, f}[x(t), y(t)]=\mathrm{FT}\left\{O_{F(5)}^{b}[x(t)] \overline{O_{F(5)}^{f}[y(t)]}\right\},
$$

which is essentially the same as Eq. (105).

The most important advantage in using the type 5 SFRFT is that it is simpler to use for fractional correlation than the original FRFT, and the performance remains the same. Because in Eq. (105) $d, h, m$, and $v$ are all set to 0 , when using the type 5 SFRFT for fractional correlation, we can save four chirp multiplication operations. And, from Eq. (111) or (112), the relationship between parameter $b$ and the width of the detection region $T$ is quite simple, and we can use $b$ to control the width of the detection region. But, for the original fractional correlation, the relationship between the parameters and the width of the detection region is complex, and it is harder in practice to use the parameters to control that width.

Some interesting properties of the type 5 SFRFT are listed here:

- Repeat of the type 5 SFRFT: 


$$
\begin{aligned}
& \qquad O_{F(5)}^{b}\left(O_{F(5)}^{b}\left\{O_{F(5)}^{b}[h(t)]\right\}\right)=h(-t) \text { any } b, \\
& \text { i.e., } \\
& \qquad O_{I F(5)}^{b}[h(u)]=O_{F(5)}^{b}\left\{O_{F(5)}^{b}[h(-u)]\right\} \quad \text { any } b \text {. }
\end{aligned}
$$

- Modulation property:

$$
O_{F(5)}^{b}[\exp (j v t) f(t)]=F_{b}(u-b v) .
$$

This property is similar to the modulation property of the original Fourier transform, except that the shift in frequency domain is magnified $b$.

- Multiplication property:

$$
O_{F(5)}^{b}[t f(t)]=j b F_{b}^{\prime}(u) .
$$

This property is similar to the multiplication of the original Fourier transform.

\section{SUMMARY AND CONCLUSIONS}

We summarize the properties of SFRFT's introduced in this paper.

- Type 1 SFRFT:

$$
F_{a}(u)=(j 2 \pi)^{-1 / 2} \int_{-\infty}^{\infty} \exp \left(-j u t+\frac{j}{2} \cot \alpha t^{2}\right) f(t) \mathrm{d} t .
$$

This equation corresponds to the LCT with parameters $\{a, b, c, d\}=\{\cot \alpha, 1,-1,0\}$. It can be used for a fractional filter implemented by a digital implementation or optical system.

- Type 2 SFRFT:

$$
\begin{aligned}
F_{a}(u)= & \left(\frac{\cot \alpha}{j 2 \pi}\right)^{1 / 2} \exp \left(-\frac{j}{2} u^{2} \cot \alpha\right) \\
& \times \int_{-\infty}^{\infty} \exp \left(-j u t \cot \alpha+\frac{j}{2} t^{2} \cot \alpha\right) f(t) \mathrm{d} t .
\end{aligned}
$$

This equation corresponds to the LCT with parameters $\{a, b, c, d\}=\{1, \tan \alpha,-2 \cot \alpha,-1\}$. It can be used for a fractional filter implemented by an optical system.

- Type 3 SFRFT:

$$
\begin{aligned}
F_{\phi}(u)= & \left(\frac{\csc \phi}{j 2 \pi w_{x}}\right)^{1 / 2} \exp \left(\frac{j}{2 w_{x}} u^{2} \cot \phi\right) \\
& \times \int_{-\infty}^{\infty} \exp \left(-j u t \frac{\csc \phi}{w_{x}}+\frac{j}{2 w_{x}} t^{2} \cot \phi\right) \\
& \times f(t) \mathrm{d} t, \quad \phi=\tan ^{-1}\left(w_{x}^{-1} \tan \alpha\right) .
\end{aligned}
$$

This equation corresponds to the LCT with parameters $\{a, b, c, d\}=\left\{\cos \phi, w_{x} \sin \phi,-\sin \phi / w_{x}, \cos \phi\right\}$. It can be used for a fractional filter implemented by a GRIN medium.

- Type 4 SFRFT:

$$
\begin{aligned}
F_{D}(u)= & \sqrt{\frac{j}{\lambda D}} \exp \left(-\frac{j k}{2 D} u^{2}\right) \int_{-\infty}^{\infty} \exp \left(\frac{j k}{D} u t+\frac{j k}{2 D} t^{2}\right) \\
& \times f(t) \mathrm{d} t, \quad D=2 \pi \tan \alpha / \lambda .
\end{aligned}
$$

This equation corresponds to the LCT with parameters $\{a, b, c, d\}=\{-1,-D / k, 2 k / D, 1\}$. It can be used for a fractional filter implemented by a radar system.

- Type 5 SFRFT:

$$
\begin{aligned}
F_{b}(u) & =O_{F(5)}^{b}[f(t)] \\
& =(j 2 \pi b)^{-1 / 2} \int_{-\infty}^{\infty} \exp \left(-\frac{j}{b} u t+\frac{j}{2 b} t^{2}\right) f(t) \mathrm{d} t .
\end{aligned}
$$

This equation corresponds to the LCT with parameters

\begin{tabular}{|c|c|c|c|c|}
\hline $\begin{array}{l}\text { Type of } \\
\text { Fourier } \\
\text { Transform }\end{array}$ & Digital Computation & Optical System & GRIN Medium & Radar System \\
\hline $\begin{array}{l}\text { Original } \\
\text { FRFT }\end{array}$ & $2 P+(P / 2) \log _{2} P$ & $\begin{array}{l}2 \text { lenses, } \\
1 \text { free space or } 1 \text { lens, } \\
2 \text { free spaces }\end{array}$ & $\begin{array}{l}\text { GRIN medium } \\
\text { with other components }\end{array}$ & 2 spherical disks, 1 free space \\
\hline $\begin{array}{l}\text { Type } 1 \\
\text { SFRFT }\end{array}$ & $P+(P / 2) \log _{2} P$ & $\begin{array}{l}\text { Same as the original } \\
\text { but only } 1 \text { component, } \\
\text { which varies with } \alpha\end{array}$ & Same as the original & Same as the original \\
\hline $\begin{array}{l}\text { Type } 2 \\
\text { SFRFT }\end{array}$ & Same as the original & 1 lens, 1 free space & Same as the original & Same as the original \\
\hline $\begin{array}{l}\text { Type } 3 \\
\text { SFRFT }\end{array}$ & Same as the original & Same as the original & GRIN medium only & Same as the original \\
\hline $\begin{array}{l}\text { Type } 4 \\
\text { SFRFT }\end{array}$ & Same as the original & Same as the original & Same as the original & 1 spherical disk, 1 plane, 1 free space \\
\hline $\begin{array}{l}\text { Type } 5 \\
\text { SFRFT }\end{array}$ & $P+(P / 2) \log _{2} P$ & Same as the original & Same as the original & Same as the original \\
\hline
\end{tabular}
$\{a, b, c, d\}=\left\{1, b,-b^{-1}, 0\right\}$. It can be used for a fractional correlation with digital implementation.

In Table 1 we show the complexity of implementation of the fractional Fourier transform (FRFT) and the simplified fractional Fourier transforms (SFRFT's) introduced in this paper. From this table we can see the advantages for each type of SFRFT. We have discussed under what conditions the linear canonical transform (LCT) will have the same effects as the original FRFT on the design of a fractional filter and fractional correlation. And we have used these results to define five types of SFRFT. These SFRFT's are simpler than the original FRFT for digital computation and optical, gradient-index medium, and

Table 1. Comparison of Implementation Complexity of Fourier-Transform Types Analyzed in This Study 
radar-system implementation. But these SFRFT's retain the same capabilities as the original FRFT for design of a fractional filter or for fractional correlation.

Our goals in this paper have been to search for the transform with the simplest implementation and to preserve the capabilities of the original FRFT. Thus, although these SFRFT's usually have no additivity properties they are useful for the practical applications. We have described five types of SFRFT. We can also derive the other types of simplified FRFT for other special applications, special implementations, or other requirements. The SFRFT's have high potential, and may replace the the original FRFT in many applications such as filter design and pattern recognition.

\section{ACKNOWLEDGMENT}

This work was supported by the National Science Council, Republic of China, under contract NSC 89-2213-E002-092.

S.-C. Pei's e-mail address is pei@cc.ee.ntu.edu.tw.

\section{REFERENCES}

1. V. Namias, "The fractional order Fourier transform and its application to quantum mechanics," J. Inst. Math. Appl. 25, 241-265 (1980).

2. L. B. Almeida, "The fractional Fourier transform and timefrequency representations," IEEE Trans. Signal Process. 42, 3084-3091 (1994).

3. M. Moshinsky and C. Quesne, "Linear canonical transformations and their unitary representations," J. Math. Phys. 12, 1772-1783 (1971)

4. K. B. Wolf, Integral Transforms in Science and Engineering (Plenum, New York, 1979), Chap. 9.

5. S. Abe and J. T. Sheridan, "Optical operations on wave functions as the Abelian subgroups of the special affine Fourier transformation," Opt. Lett. 19, 1801-1803 (1994).

6. L. M. Bernardo, " $A B C D$ matrix formalism of fractional Fourier optics,” Opt. Eng. 35, 732-740 (1996).
7. H. M. Ozaktas and D. Mendlovic, "Every Fourier optical system is equivalent to consecutive fractional-Fourierdomain filtering," Appl. Opt. 35, 3167-3170 (1996).

8. S. C. Pei and J. J. Ding, "Closed form discrete fractional and affine Fourier transforms," IEEE Trans. Signal Process. 48, 1338-1353 (2000).

9. H. M. Ozaktas, O. Arikan, M. A. Kutay, and G. Bozdagi, "Digital computation of the fractional Fourier transform," IEEE Trans. Signal Process. 44, 2141-2150 (1996).

10. A. M. Almanasreh and M. G. Abushagur, "Fractional correlation based on the modified fractional order Fourier transform," Opt. Eng. 37, 175-184 (1998).

11. M. A. Kutay, H. M. Ozaktas, O. Arikan, and L. Onural, "Optimal filter in fractional Fourier domains," IEEE Trans. Signal Process. 45, 1129-1143 (1997).

12. A. W. Lohmann, D. Mendlovic, and Z. Zalevsky, "Fractional Hilbert transform," Opt. Lett. 21, 281-283 (1996).

13. H. M. Ozaktas, B. Barshan, D. Mendlovic, and L. Onural, "Convolution, filtering, and multiplexing in fractional Fourier domains and their rotation to chirp and wavelet transform,” J. Opt. Soc. Am. A 11, 547-559 (1994).

14. S. C. Pei and J. J. Ding, "Relations between the fractional operations and the Wigner distribution, ambiguity function," submitted to IEEE Trans. Signal Process.

15. L. B. Almeida, "Product and convolution theorems for the fractional Fourier transform," IEEE Signal Process. Lett. 4, 15-17 (1997)

16. A. I. Zayed, "A convolution and product theorem for the fractional Fourier transform," IEEE Signal Process. Lett. 5, 101-103 (1998).

17. B. Barshan, M. A. Kutay, and H. M. Ozaktas, "Optimal filtering with linear canonical transformations," Opt. Commun. 135, 32-36 (1997).

18. L. Yu, M. Huang, L. Wu, Y. Lu, W. Huang, M. Chen, and Z. Zhu, "Fractional Fourier transform and the elliptic gradient-index medium," Opt. Commun. 152, 23-25 (1998).

19. P. Pellat-Finet and G. Bonnet, "Fractional order Fourier transform and Fourier optics," Opt. Commun. 111, 141-154 (1994).

20. D. Mendlovic, H. M. Zalevsky, and A. W. Lohmann, "Fractional correlation," Appl. Opt. 34, 303-309 (1995).

21. A. W. Lohmann, Z. Zalevsky, and D. Mendlovic, "Synthesis of pattern recognition filters for fractional Fourier processing," Opt. Commun. 128, 199-204 (1996).

22. S. Granieri, R. Arizaga, and E. E. Sicre, "Optical correlation based on the fractional Fourier transform," Appl. Opt. 36, 6636-6645 (1997) 